\title{
G.O.H Naumann, LM Holbach, F.E. Kruse: Applied Pathology for Ophthalmic Microsurgeons
}

\author{
Springer, ISBN 978-3-540-24189-8, Stephen J. Ryan, M.D. and Narsing A. Rao, M.D., \\ Doheny Eye Institute, Los Angeles, California
}

\section{Stephen Ryan}

Published online: 18 June 2008

(C) Springer-Verlag 2008

Naumann, Holbach, and Kruse's profusely illustrated book, Applied Pathology for Ophthalmic Microsurgeons, conveys the basic concepts of microsurgical intervention in the management of various ophthalmic disorders, based on anatomic principles and on the histopathological changes that affect the ocular structures. The highlight of the book is its documentation of the concepts. The excellent illustrations include the surgical anatomy of ophthalmic structures that are affected by pathologic changes, as well as typical histopathologic changes, all displayed in vivid color. The book also provides a concise listing of common ocular diseases that may require surgical intervention.

Intraocular surgery is performed with the operating microscope; thus, microsurgery is the rule for ophthalmic surgeons. A thorough understanding of pathologic structures and of the pathophysiology of disease is essential for ophthalmic surgeons. Thus, this book fills an important need.

This well-written book details the histopathological changes that occur with various ophthalmic diseases, and provides for a clear understanding of the necessary surgical interventions. Each of the easily read chapters begins with an introduction and a description of the essential physiologic functions of the tissues. This is followed by details of the surgical anatomy required for microsurgical intervention. The surgical pathology and frequency of various clinical and pathologic entities affecting the structure are highlighted and summarized in tables. Each chapter includes a display of the histopathology with classic stains, supplemented with immunohistochemical findings and ultrastruc-

\section{S. Ryan $(\bowtie)$}

Section: RE, Ophthalmology, State University of Londrina,

1450 San Pablo St,

Los Angeles, CA 90033-4681, USA

e-mail: sryan@doheny.org tural features of neoplastic disorders. Relevant pre-surgical clinical illustrations, the gross appearance of surgically excised specimens, and pertinent clinicopathologic correlations are seen throughout the book. Microsurgical interventions, such as current procedures used to manage glaucoma, are clearly described. Indications and contraindications for the procedures are described, primarily based on the disease processes, the anatomic principles, and the natural history of the disease. Furthermore, the chapters cover common systemic diseases that can affect the management of ophthalmic disorders during intraocular surgery, including diabetes, hypertension, infectious diseases, hematologic disorders, and pseudoexfoliation syndrome. Although infections and hematologic disorders are only briefly mentioned, the chapter on pseudoexfoliation syndrome provides clinical pearls to help the physician understand and anticipate possible complications. Pertaining to the management of pseudoexfoliation, the book states "regarding the clinical management of these patients, the importance of early recognition of the pathologic features, expectations of a higher complication rate, close attention to postoperative follow-up and well-informed patient before surgery have to be emphasized" (page 375).

At the end of the book is a listing of figures with brief legends. This unique feature is highly useful for quick reference to the clinical and histologic features of ophthalmic diseases. Ophthalmic microsurgeons, ophthalmic pathologists, and graduate students pursuing training in ophthalmology will benefit from reviewing these chapters, particularly to develop a clear understanding of the principles underlying the microsurgical approach to managing ophthalmic diseases. The authors have succeeded in delineating the principles of microsurgical intervention, using relevant, high-quality illustrations supplemented with interesting diagrams and tables. 